\title{
Monitoring rejection after heart transplantation: cytoimmunological monitoring on blood cells and quantitative birefringence measurements on endomyocardial biopsy specimens
}

\begin{abstract}
P L J Wijngaard, J A Gimpel, H-J Schuurman, A van der Meulen, F H J Gmelig Meyling, G Jambroes
\end{abstract}

\begin{abstract}
Cytoimmunological monitoring and quantitative birefringence measurements were used as potential aids in diagnosing acute rejection after heart transplantation instead of histopathological assessment of the endomyocardial biopsy specimen alone. Cytoimmunological monitoring was based on morphological inspection and quantitation of mononuclear cells, particularly activated lymphoid cells. Quantitative birefringence measurements comprise a variable for myocyte contractile function. Its read out is the ratio of the degree of birefringence before contraction to that after. Cytoimmunological monitoring indicated significantly higher concentrations of activated lymphocytes in moderate or severe acute rejection, and quantitative birefringence measurements indicated decreased myocyte function during severe and resolved or resolving rejection. Cytoimmunological monitoring and quantitative birefringence measurements were diagnostically most useful in terms of sensitivity, specificity, and predictive value, when only data gathered before the first episode of acute rejection were considered. For cytoimmunological monitoring, diagnostic relevance was optimal when the data were expressed as relative proportions of activated lymphocytes. The quantitative birefringence measurements correlated best with analysis of the endomyocardial biopsy specimen when a cut off value of 1.25 was used. When both methods for diagnosing acute rejection were analysed together, no improvement in sensitivity (value 0.44 ) was found, but the specificity increased to 0.98 and the predictive value to about 0.80 .

It is concluded that cytoimmunological monitoring is a useful, non-invasive additional method for diagnosing the first period of acute rejection after heart transplantation and that quantitative birefringence measurements give valuable information on the extent of myocyte damage.
\end{abstract}

Acute rejection is one of the first life threatening complications to occur after heart trans- plantation. The diagnosis of acute rejection is based primarily on histopathological assessment of the endomyocardial biopsy specimen. ${ }^{1}$ New methods for the diagnosis of acute rejection have now been introduced..$^{2-5}$ Noninvasive cytoimmunological monitoring is based on morphological inspection of mononuclear cells combined with phenotype assessment of lymphocyte subpopulations. ${ }^{6-11}$ The results of cytoimmunological monitoring, that is, the determination of the number of activated lymphocytes, permits the differentiation between graft acceptance, rejection, and infection (table 1). ${ }^{9}$ To estimate myocyte contractile function, quantitative birefringence measurements were performed on endomyocardial biopsy specimens. Quantitative birefringence measurements give a quantitative assessment of both the relaxation of the myocytes and their ability to contract after the addition of adenosine triphosphate and calcium. Quantitative birefringence measurements are a reliable monitor for myocardial protection during cardiopulmonary bypass surgery and give an indication of the postoperative condition of the patient. ${ }^{12-14}$ In assessing the quality of donor hearts for heart transplantation, quantitative birefringence measurements also provide reliable indices of the graft's viability and its functional state. ${ }^{15}$

In our heart transplantation programme, cytoimmunological monitoring and quantitative birefringence measurements were introduced to diagnose acute rejection. We compared these methods with the histopathological assessment of the endomyocardial biopsy specimens. The diagnostic usefulness of both assays was expressed in terms of sensitivity, specificity, and predictive value. For cytoimmunological monitoring we determined the best way to express the data-that is, relative proportions $v$ absolute numbers of activated lymphocytes - and the results of the standard immunosuppressive treatment were registered accordingly. Furthermore, a correlation between cytoimmunological results expressed in relative proportions and absolute numbers and the period after transplantation during which cytoimmunological monitoring could best be used was analysed. We measured the quantitative birefringence measurements of biopsy specimens to compare the outcome of histopathological assessment in terms of rejection (Billingham scale ${ }^{1}$ ) with a functional variable. 
Table 1 Cytoimmunological monitoring criteria for diagnosis of acute rejection and infection

\begin{tabular}{|c|c|c|c|c|}
\hline & \multirow[b]{2}{*}{ Normal } & \multirow[b]{2}{*}{ Rejection } & \multicolumn{2}{|l|}{ Infection } \\
\hline & & & Viral & Bacterial \\
\hline $\begin{array}{c}\text { Activation } \\
\text { Relative } \\
\text { Absolute }\end{array}$ & $\begin{array}{l}<5 \% \\
<50 / \mu 1\end{array}$ & $\begin{array}{l}>5 \% \\
>50 / \mu \mathrm{l}\end{array}$ & $\begin{array}{l}>5 \% \\
>50 / \mu \mathrm{l}\end{array}$ & $\begin{array}{l}>5 \% \\
>50 / \mu \mathrm{l}\end{array}$ \\
\hline $\begin{array}{l}\text { Large granular lymphocytes } \\
\text { Relative } \\
\text { Absolute } \\
\text { CD4:CD8 ratio } \\
\text { Leu- } 7^{+} \text {cells } \\
\text { Young myeloid cells in blood smear }\end{array}$ & $\begin{array}{l}<15 \% \\
<150 / \mu \mathrm{l} \\
>1 \cdot 0 \\
<15 \% \\
\text { No }\end{array}$ & $\begin{array}{l}<15 \% \\
<150 / \mu \mathrm{l} \\
>1 \cdot 0 \\
<15 \% \\
\text { No }\end{array}$ & $\begin{array}{l}>15 \% \\
>150 / \mu \mathrm{l} \\
<1.0 \\
>15 \% \\
\text { No }\end{array}$ & $\begin{array}{l}<15 \% \\
<150 / \mu 1 \\
>1.0 \\
<15 \% \\
\text { Yes }\end{array}$ \\
\hline
\end{tabular}

\section{Methods}

The study comprised 16 patients who underwent orthotopic heart transplantation. Donor and recipient were matched for $\mathrm{ABO}$ blood group antigens but not for HLA histocompatibility. A negative cross match between patient serum and donor leucocytes was also needed. As a standard immunosuppressive regimen the patients received either cyclosporine and prednisolone (double combination treatment) (cases 1-9) or cyclosporine, prednisolone, and azathioprine (triple combination treatment) (cases 10-16). Acute rejection (grade 2 or 3 ), as defined by histopathological assessment of the endomyocardial biopsy specimen, ${ }^{1}$ was treated with a methylprednisolone pulse for three days, or with rabbit anti-thymocyte globulin or both. Cytoimmunological monitoring was performed almost daily during the first postoperative period. Thereafter, it was performed at all outpatient visits until six months after transplantation, and later on every day that an endomyocardial biopsy specimen was taken. Quantitative birefringence measurements were performed on sections of almost every endomyocardial biopsy specimen. The follow up of 16 patients ranged from 78 to 775 days; a total of 185 endomyocardial biopsy specimens was available for our study, out of which 122 showed no signs of acute rejection (grade 0 ), 15 slight rejection (grade 1), 22 moderate rejection (grade 2), 13 severe rejection (grade 3) and 13 endomyocardial biopsy specimens resolved or resolving rejection (grade 4). In 164 cases cytoimmunological monitoring and quantitative birefringence measurements were performed on the same day as the endomyocardial biopsy specimen was taken. Cytoimmunological monitoring and quantitative birefringence measurements were performed on the same day in 143 cases.

\section{CYTOIMMUNOLOGICAL MONITORING}

Mononuclear cells were isolated from venous blood samples treated with heparin by FicollIsopaque density gradient centrifugation. Cytocentrifuge preparations (75000 cells/ slide) were stained with May-Grünwald Giemsa. The lymphoid cells were differentiated into small resting lymphocytes, activated lymphocytes, lymphoblasts, plasmacytoid cells and large granular lymphocytes. ${ }^{9}$ Furthermore, a total and a differential whole blood leucocyte count were performed. Data were expressed in relative proportions as the percentage of acti- vated cells (lymphoblasts, activated lymphocytes, and plasmacytoid cells) within the total lymphoid cell population, and in absolute numbers as the number of activated cells per microlitre of blood. The absolute numbers were calculated using the percentage of lymphocytes in the differential leucocyte count, the total leucocyte count per microlitre of blood, and the percentages of lymphoid cells in the mononuclear cell isolate. We adapted decision criteria from the literature, ${ }^{67}$ such as activated lymphocytes exceeding $5 \%$ for the relative cytoimmunological monitoring and exceeding 50 cells $/ \mu$ l blood for the absolute cytoimmunological monitoring which were regarded as indicating lymphocyte activation.

Phenotyping of lymphocytes was performed either on cytocentrifuge preparations by an indirect immunoperoxidase staining method ${ }^{4}$ or in suspension by an indirect immunofluorescence technique with analysis by a FACS analyser (Cytofluorograf 50-H, Ortho Diagnostic systems, Westwood, Massachusetts, USA). Monoclonal antibodies used were antiLeu-2a (CD8), anti-Leu-3(a + b) (CD4), and anti-Leu-7 (natural killer cells) (all from Becton-Dickinson, Mountain View, California, USA). The lymphocyte subpopulations were expressed as percentages of total lymphoid cells and the CD4: CD8 ratio was calculated.

\section{QUANTITATIVE BIREFRINGENCE MEASUREMENTS}

Endomyocardial biopsy was performed with the use of a Bright bioptome. The biopsy specimens sized about $1 \mathrm{~mm}^{3}$ were expelled on to a swab and immediately chilled by immersion in a $\mathrm{n}$-hexane at $-70^{\circ} \mathrm{C}$ for about 30 seconds, followed by storage at $-70^{\circ} \mathrm{C}$ until analysis.

Frozen tissue specimens were mounted on metal chucks by means of a drop of water and sectioned at $8 \mu \mathrm{m}$ at $-37^{\circ} \mathrm{C}$. A freshly mounted section was examined in air under a Zeiss Universal polarising microscope, with the polariser and the analyser in the crossed position. The longitudinal muscle fibres were positioned in the centre of the microscopic field and rotated in such a way that they were lying at a $45^{\circ}$ angle to the $\mathrm{X}$ and $\mathrm{Y}$ axis. $\mathrm{A}$ lambda/30 Brace-Köhler rotating compensator was introduced between the specimen and the analyser. The compensator was then rotated until the myofibrils just seemed to go black. The compensator was read and a drop of sodium barbitone buffer ( $\mathrm{pH} \mathrm{8.0)}$ containing 2 $\mathrm{mmol} / 1$ adenosinetriphosphate (ATP) and 36 $\mathrm{mmol} / 1 \mathrm{CaCl}_{2}$ was added to the section and the immediate increase in the birefringence caused by the contraction of the myofibrils was measured again. The increase in birefringence is converted into a difference in linear optical path. From these numbers the birefringence ratio (optical path difference caused by ATP + calcium divided by initial optical path difference) was calculated. Because both measurements are performed at the same myofibrils for the birefringence ratio there is no need to correct for the thickness of the sections-that is, myofibril concentration. We 
used two cut off values for distinguishing acute rejection (decreased quantitative birefringence measurements values): a birefringence assessment of 1.25 , which was the mean value in endomyocardial biopsy specimens without acute rejection (grade 0 ) $-2 \times S E M$ and $1 \cdot 13$, which was the mean value in endomyocardial biopsy specimens with severe acute rejection (grade 3 ) $+2 \times$ SEM.

Cytoimmunological monitoring data, expressed in relative proportions, and quantitative birefringence measurement data were analysed with respect to the different histopathological grades of the endomyocardial biopsy specimens according to Billingham. ${ }^{1}$ For individual patients the correlation between cytoimmunological monitoring results expressed in relative proportions and expressed in absolute numbers was calculated. The diagnostic usefulness was expressed in terms of sensitivity, specificity, and predictive value with histopathology of the endomyocardial biopsy specimen as reference: sensitivity is calculated as true positive results/(true positives + false negatives), specificity as true negative results/ (true negatives + false positives), and predictive value as true positive results/(true positives + false positives). In these calculations cytoimmunological monitoring and quantitative birefringence measurement data were categorised for three groups: group A comprised all data from day 1 after transplantation; group B comprised the data from day 1 until the day of the first endomyocardial biopsy specimen that manifested acute rejection (grade 2-3), and group C comprised the data with exclusion of those during ongoing and resolved or resolving rejection. Ongoing rejection is defined as endomyocardial biopsy specimens with grade 2 or 3 rejection during or directly after treatment for rejection. In this way the effects of additional immunosuppressive treatment for episodes of acute rejection could be analysed.

The parametric Student's $t$ test, the nonparametric $U$ test, the Pearson correlation coefficient, and the analysis of variance (ANOVA) were applied.

\section{Results}

\section{CYTOIMMUNOLOGICAL MONITORING AND} QUANTITATIVE BIREFRINGENCE MEASUREMENT DATA IN RELATION TO HISTOPATHOLOGY OF ENDOMYOCARDIAL BIOPSY SPECIMENS

The results of cytoimmunological monitoring expressed in relative proportions and quantitative birefringence measurements in groups according to histopathological assessment of the endomyocardial biopsy specimens are presented in figs 1 and 2. Cytoimmunological monitoring data indicating infection were excluded in fig 1 . Significant differences among groups according to histopathology were observed for cytoimmunological monitoring and quantitative birefringence measurements (ANOVA, $p<0.001$ ). In comparing individual groups, significance was calculated for cytoimmunological monitoring between grade 2 and 0 , and between grade 2 and 1 $(t$ test, $\mathrm{p}<0.05)$. For quantitative birefrin-

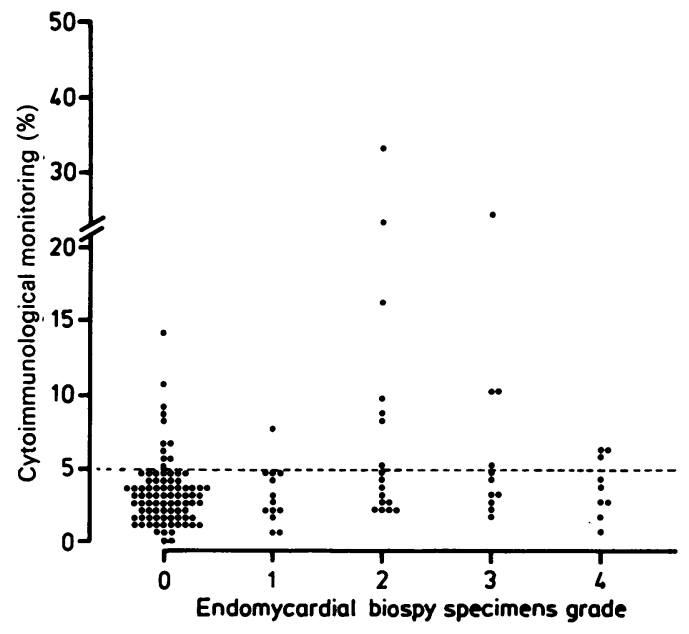

Figure 1 Relation of cytoimmunological monitoring, expressed in relative proportions of activated lymphocytes, with histopathological assessment of endomyocardial biopsy specimens (grading according to Billingham). Data exclude those in which cytoimmunological monitoring indicated infection. The decision level of $5 \%$ is also indicated. The differences between the groups according to histopathology are significant (ANOVA, $p<0.001)$.

gence measurements, values for grade 3 rejection were significantly lower than for grade $0(t$ test, $\mathrm{p}<0.001$ ), 1 , and 2 ( $t$ test, $\mathrm{p}<0.05$ ); values for grade 4 were significantly lower than for grade 1 ( $t$ test, $\mathrm{p}<0.05$ ) and 0 ( $t$ test, $\mathrm{p}<$ $0.01)$.

Data were also analysed for combination of groups-in case these groups showed no significant differences. For cytoimmunological

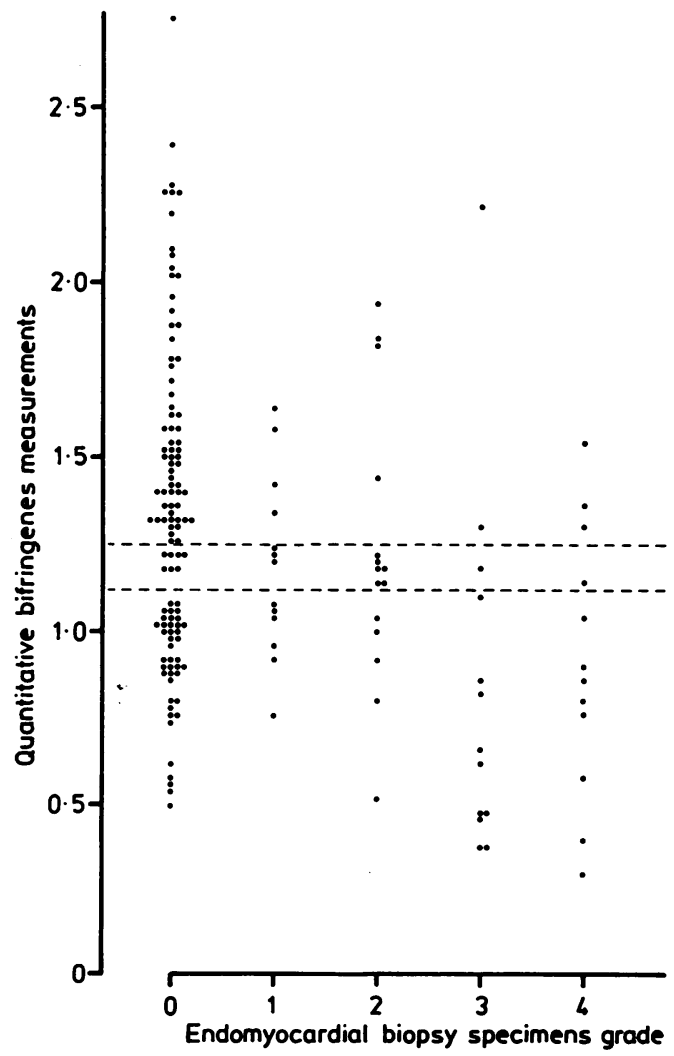

Figure 2 The relation of the quantitative birefringence measurement data with the histopathology of the endomyocardial biopsy specimens (grading according to Billingham). The cut off values used in concluding rejection are also indicated. The differences in quantitative birefringence measurements between the groups according to histopathology are significant (ANOVA,p $<0.001)$. 
Table 2 Coefficient of correlation between cytoimmunological monitoring data expressed in absolute and relative numbers

\begin{tabular}{|c|c|c|c|c|c|c|}
\hline Case No & Group $A$ & $(n=)$ & Group B & $(n=)$ & Group $C$ & $(n=)$ \\
\hline \multicolumn{7}{|c|}{ Double treatment : } \\
\hline 1 & $0.85^{\star}$ & (43) & $0.94^{\star}$ & (21) & 0.91 夫 & $(30)$ \\
\hline 2 & $0 \cdot 65^{\star}$ & (41) & $0 \cdot 76^{\star}$ & (25) & $0 \cdot 76^{\star}$ & $(25)$ \\
\hline 3 & $0 \cdot 30$ & (69) & $0.98^{\star}$ & (5) & $0 \cdot 50^{\star}$ & (37) \\
\hline 4 & $0 \cdot 86^{\star}$ & (36) & $0 \cdot 86^{\star}$ & (36) & $0 \cdot 86^{\star}$ & $(36)$ \\
\hline 5 & $0.96^{\star}$ & (9) & $0.96^{\star}$ & (9) & $0.96^{\star}$ & (9) \\
\hline 6 & $0 \cdot 84^{\star}$ & (43) & $0.95^{\star}$ & (7) & $0.81^{\star}$ & (30) \\
\hline 7 & $0.96^{\star}$ & (27) & $0.96^{\star}$ & (27) & $0.96^{\star}$ & (27) \\
\hline 8 & $0 \cdot 50^{\star}$ & (36) & $0.93^{\star}$ & (10) & 0.39 & $(22)$ \\
\hline 9 & 0.36 & (34) & $0.96^{\star}$ & (6) & $0 \cdot 27$ & $(20)$ \\
\hline \multicolumn{7}{|c|}{ Triple treatment: } \\
\hline 10 & 0.57 & (9) & 0.61 & (4) & 0.61 & (4) \\
\hline 11 & 0.40 & (10) & 0.40 & (10) & $0 \cdot 40$ & (10) \\
\hline 12 & $0.59^{\star}$ & $(46)$ & $0 \cdot 74^{\star}$ & (24) & $0.58^{\star}$ & (39) \\
\hline 13 & $0.97^{\star}$ & (7) & $0.97^{\star}$ & (7) & $0.97^{\star}$ & (7) \\
\hline 14 & $0 \cdot 64^{\star}$ & (59) & 0.63 & (8) & $0 \cdot 70$ & (35) \\
\hline 15 & 0.37 & (28) & 0.37 & (28) & 0.37 & (28) \\
\hline 16 & $0 \cdot 28$ & (25) & $0 \cdot 82^{\star}$ & (16) & $0 \cdot 28$ & (21) \\
\hline
\end{tabular}

monitoring this was done for grades 0 and 1 (mean 3.34, SEM 0.22) and for grades 2 and 3 (mean 7.39, SEM 1.50); the difference between these was significant $(t$ test, $\mathrm{p}<0.001)$. For quantitative birefringence measurements this was done for grades 1 and 2 (mean 1.21, SEM $0 \cdot 06$ ). This value was significantly higher than that in grade 3 (mean 0.85 , SEM 0.14 ) and in grade 4 (mean 0.92, SEM 0.11) ( $t$ test, p < 0.001 ).

The correlation coefficients $(r)$ for the cytoimmunological monitoring results expressed in relative proportions and in absolute numbers are given in table 2 for each individual patient. These were calculated for all data (denoted as group A), for data till the first episode of acute rejection (group B), and for the data excluding those obtained during ongoing and resolved or resolving rejection (group C). The highest values were found for group B in cases 1-9. These patients who were receiving double combination immunosuppression treatment, showed significantly higher values than cases 10-16 who were receiving triple combination treatment ( $U$ test, $p<0.05$ ).

The sensitivity, specificity, and predictive values for diagnosing acute rejection are given in table 3 for cytoimmunological monitoring and in table 4 for quantitative birefringence monitoring. Cytoimmunological monitoring expressed in relative proportions showed the highest sensitivity $(0.58)$, specificity $(0.91)$, and

Table 3 Diagnostic usefulness of cytoimmunological monitoring, expressed in either relative proportions or in absolute numbers, for diagnosing acute rejection

\begin{tabular}{|c|c|c|c|c|c|}
\hline $\begin{array}{l}\text { Cytoimmunological } \\
\text { monitoring }\end{array}$ & Group & $(n=)$ & Sensitivity & Specificity & Predictive value \\
\hline \multicolumn{6}{|c|}{ Relative cytoimmunological monitoring (cut off value $5^{\circ}$ ) } \\
\hline $\begin{array}{l}\text { All data } \\
\text { Infection } \\
\text { All data } \\
\text { Infection } \\
\text { All data } \\
\text { Infection }\end{array}$ & $\begin{array}{l}\text { A } \\
\text { A } \\
\text { B } \\
\text { B } \\
\text { C } \\
\text { C }\end{array}$ & $\begin{array}{r}(151) \\
(128) \\
(59) \\
(58) \\
(137) \\
(117)\end{array}$ & $\begin{array}{l}0.48 \\
0.39 \\
0.58 \\
0.55 \\
0.47 \\
0.41\end{array}$ & $\begin{array}{l}0.75 \\
0.88 \\
0.91 \\
0.91 \\
0.75 \\
0.88\end{array}$ & $\begin{array}{l}0.35 \\
0.48 \\
0.64 \\
0.60 \\
0.23 \\
0.37\end{array}$ \\
\hline \multicolumn{6}{|c|}{ Absolute cytoimmunological monitoring (cut off value $50 / \mu l$ blood) } \\
\hline $\begin{array}{l}\text { All data } \\
\text { Infection } \\
\text { All data } \\
\text { Infection } \\
\text { All data } \\
\text { Infection }\end{array}$ & $\begin{array}{l}\text { A } \\
\text { A } \\
\text { B } \\
\text { B } \\
\text { C } \\
\text { C }\end{array}$ & $\begin{array}{r}(151) \\
(139) \\
(59) \\
(58) \\
(137) \\
(126)\end{array}$ & $\begin{array}{l}0.36 \\
0.30 \\
0.50 \\
0.45 \\
0.42 \\
0.35\end{array}$ & $\begin{array}{l}0.84 \\
0.91 \\
0 \cdot 87 \\
0 \cdot 87 \\
0 \cdot 84 \\
0.91\end{array}$ & $\begin{array}{l}0.39 \\
0.47 \\
0.50 \\
0.45 \\
0.30 \\
0.38\end{array}$ \\
\hline
\end{tabular}

predictive value $(0.64)$ when all data up to the first acute rejection were considered (group B). Lower values were calculated when all data (group A) and all data excluding those obtained during ongoing and resolved or resolving rejection (group C) were considered. Cytoimmunological monitoring expressed in absolute numbers per $\mu \mathrm{l}$ blood showed lower values than data expressed in relative numbers. When cytoimmunological monitoring data indicating infection were excluded from the calculations, a lower sensitivity and higher specificity were calculated for all three groups.

Quantitative birefringence measurement data were analysed with respect to two cut off values: 1.25 (mean quantitative birefringence measurement values of grade $2 \times \mathrm{SEM}$ ) and 1.13 (mean quantitative birefringence measurement values of grade $3+2 \times$ SEM) (fig 2). The highest values (sensitivity 0.90 , specificity 0.60 , and predictive value 0.32 ) were calculated using 1.25 as cut off value and when only data up to the first episode of acute rejection (group B) were considered. When 1.13 was taken as the cut off value, a lower sensitivity and predictive value but a higher specificity were calculated.

Finally, we calculated sensitivity, specificity, and predictive value for cytoimmunological monitoring and quantitative birefringence measurements data combined (table 5). In this analysis highest values were also found for data up to the first episode of acute rejection (group B). In this group highest values were calculated for all data which included cytoimmunological monitoring data indicating infection and for a cut off value of 1.25 in quantitative birefringence measurements (sensitivity 0.50 , specificity 0.98 , and predictive value 0.83 ). The same specificity was calculated for a cut off value of 1.13 in quantitative birefringence measurements and considering cytoimmunological monitoring data excluding those indicating infection, but sensitivity and predictive value were lower.

\section{Discussion}

Cytoimmunological monitoring has been proposed as a simple and non-invasive technique for the diagnosis of acute rejection after heart transplantation. ${ }^{6-9}$ In this study cytoimmunological monitoring data, expressed in relative proportions, correlated better with absolute numbers when the patients were receiving double combination immunosuppressive treatment (cyclosporine and prednisone) than when the patients were receiving triple combination treatment (with azathioprine) (table 2). The lower correlation coefficients in cases of triple treatment may be explained by differences in susceptibility of lymphocyte subsets (defined by morphology associated with cell activation) to azathioprine. Relative and absolute cytoimmunological monitoring data also correlated well before the first episode of acute rejection, but not subsequently. Obviously, additional treatment begun after the first period of rejection affects activated and other lymphocytes in a different 
Table 4 Diagnostic usefulness of quantitative birefringence measurements for diagnosing acute rejection

\begin{tabular}{llllll}
\hline Cut off value & Groups & $(n=)$ & Sensitivity & Specificity & Predictive value \\
\hline 1.25 & A & $(152)$ & 0.79 & 0.54 & 0.28 \\
1.13 & A & $(152)$ & 0.54 & 0.62 & 0.24 \\
1.25 & B & $(57)$ & 0.90 & 0.60 & 0.32 \\
1.13 & B & $(57)$ & 0.60 & 0.66 & 0.27 \\
1.25 & C & $(142)$ & 0.78 & 0.54 & 0.20 \\
1.13 & C & $(142)$ & 0.50 & 0.62 & 0.16 \\
\hline
\end{tabular}

way, resulting in lower values of correlation coefficients between absolute and relative proportions. Interestingly, we observed that most patients showed an increase in percentages of activated lymphocytes but a decrease in the absolute number of total lymphocytes when additional immunosuppression treatment was started.

Cytoimmunological monitoring shows significantly higher percentages of activation for endomyocardial biopsy specimens with moderate or severe rejection than for endomyocardial biopsy specimens with no or mild rejection (fig 1). The sensitivity, specificity, and predictive value of cytoimmunological monitoring in diagnosing acute rejection were highest for data obtained up to the first episode of acute rejection expressed in relative proportions. Deletion of cytoimmunological monitoring data indicating infection did not improve these variables of diagnostic relevance. These variables were even lower when all data with or without those of ongoing and resolved or resolving rejection were considered. This may be explained by two phenomena: firstly, episodes of acute rejection after the first one are less "acute" and may not be reflected by increased numbers of activated lymphocytes in the circulation; secondly, in the postoperative period infections can occur which are reflected in lymphocyte activation without an endomyocardial biopsy specimen indicating rejection. We conclude that cytoimmunological monitoring should be used until the first episode of acute rejection; thereafter it has little value in the diagnosis of acute rejection. In addition to our conclusion that cytoimmunological monitoring should be used until the patient sustains the first episode of acute rejection, we suggest that this occurs during the first six months after transplantation. Subse-

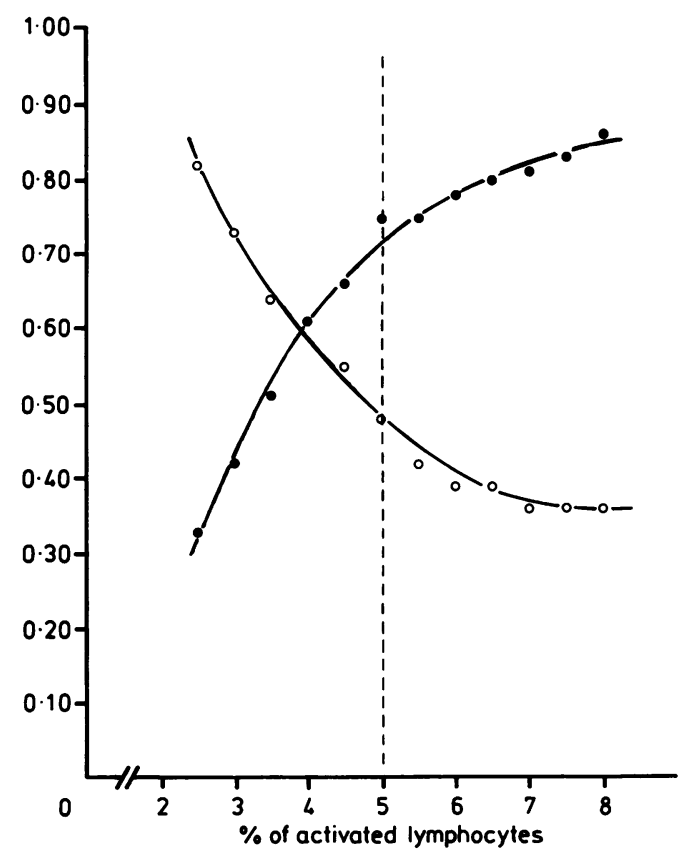

Figure 3 Diagnostic usefulness, expressed in sensitivity (O) and specificity ( $)$, of cytoimmunological monitoring expressed in relative proportions as function of the cut off values of activated lymphocytes. This was calculated for total data from day 1 after transplantation (group A). The 5\% level used in this study is also indicated.

quently, cytoimmunological monitoring is less helpful. Our own data supported this phenomenon, but are not presented as the number of observations were too few to warrant statistical analysis. These conclusions have also been reached by others (Hammer et al, personal communication). This does not exclude the potential value of cytoimmunological monitoring in diagnosing infection (table 1), but this is beyond the scope of this article. Furthermore, cytoimmunological monitoring cannot be used as the sole variable in diagnosing acute rejection. It has insufficient sensitivity to assess all cases of acute rejection and its predictive value is not high enough either. This is obviously related to the decision value applied to interpretation. Taking lower percentages of activated cells as the cut off point, the sensitivity increases with decreasing specificity (fig 3).

Table 5 Diagnostic usefulness of cytoimmunological monitoring, expressed in relative proportions and quantitative birefringence measurements for diagnosing rejection

\begin{tabular}{|c|c|c|c|c|c|c|}
\hline & \multicolumn{2}{|l|}{ Cut off values } & \multirow[b]{2}{*}{$(n=)$} & \multirow[b]{2}{*}{ Sensitivity } & \multirow[b]{2}{*}{ Specificity } & \multirow[b]{2}{*}{$\begin{array}{l}\text { Predictive } \\
\text { value }\end{array}$} \\
\hline & $\begin{array}{l}\text { Cytoimmunological } \\
\text { monitoring }\end{array}$ & $\begin{array}{l}\text { Quantitative birefringence } \\
\text { measurements }\end{array}$ & & & & \\
\hline \multicolumn{7}{|l|}{ Group A: } \\
\hline $\begin{array}{l}\text { All data } \\
\text { Infection } \\
\text { All data } \\
\text { Infection }\end{array}$ & $\begin{array}{l}5^{\circ}{ }_{0} \\
5^{\circ}{ }_{o} \\
5^{\circ}{ }_{0} \\
5^{\circ}{ }_{0}\end{array}$ & $\begin{array}{l}1 \cdot 25 \\
1 \cdot 25 \\
1 \cdot 13 \\
1 \cdot 13\end{array}$ & $\begin{array}{l}(131) \\
(113) \\
(131) \\
(113)\end{array}$ & $\begin{array}{l}0 \cdot 25 \\
0 \cdot 23 \\
0 \cdot 23 \\
0 \cdot 18\end{array}$ & $\begin{array}{l}0.91 \\
0.97 \\
0.91 \\
0.97\end{array}$ & $\begin{array}{l}0.50 \\
0.71 \\
0.40 \\
0.67\end{array}$ \\
\hline \multicolumn{7}{|l|}{ Group B: } \\
\hline $\begin{array}{l}\text { All data } \\
\text { Infection } \\
\text { All data } \\
\text { Infection }\end{array}$ & $\begin{array}{l}5^{\circ} \% \\
5^{\circ} \% \\
5^{\circ} \% \\
5^{\circ} \%\end{array}$ & $\begin{array}{l}1 \cdot 25 \\
1.25 \\
1 \cdot 13 \\
1 \cdot 13\end{array}$ & $\begin{array}{l}(50) \\
(49) \\
(50) \\
(49)\end{array}$ & $\begin{array}{l}0 \cdot 50 \\
0 \cdot 44 \\
0 \cdot 30 \\
0 \cdot 33\end{array}$ & $\begin{array}{l}0.98 \\
0.98 \\
0.98 \\
0.98\end{array}$ & $\begin{array}{l}0.83 \\
0.80 \\
0.75 \\
0.75\end{array}$ \\
\hline \multicolumn{7}{|l|}{ Group C: } \\
\hline $\begin{array}{l}\text { All data } \\
\text { Infection } \\
\text { All data } \\
\text { Infection }\end{array}$ & $\begin{array}{l}5^{\circ} \% \\
5^{\circ} \% \\
5^{\circ} \% \\
5_{0}^{\circ}\end{array}$ & $\begin{array}{l}1 \cdot 25 \\
1 \cdot 25 \\
1 \cdot 13 \\
1 \cdot 13\end{array}$ & $\begin{array}{l}(121) \\
(105) \\
(121) \\
(105)\end{array}$ & $\begin{array}{l}0 \cdot 25 \\
0 \cdot 23 \\
0 \cdot 23 \\
0 \cdot 18\end{array}$ & $\begin{array}{l}0.91 \\
0.97 \\
0.91 \\
0.97\end{array}$ & $\begin{array}{l}0.50 \\
0.71 \\
0.40 \\
0.67\end{array}$ \\
\hline
\end{tabular}


Quantitative birefringence measurements are a sensitive indicator for myocardial contractile ability. ${ }^{1314}$ They are significantly lower in endomyocardial biopsy specimens with grade 3 rejection; endomyocardial biopsy specimens with grades 1 and 2 rejection show values in between the values for grades 0 and 3 (fig 2). Apparently, the myocyte contractile ability is only affected in grade 3-notably, the histological diagnosis of mild rejection is merely based on lymphocyte infiltration and not on myocyte damage. Although the average values of quantitative birefringence measurements differ significantly for the different endomyocardial biopsy specimen grades, we found a huge overlap between the groups defined by histopathological analysis. This impairs the diagnostic usefulness of quantitative birefringence measurements, which is restricted to the assessment of episodes of severe rejection (grade 3). In terms of clinical outcome this has little relevance as grades 2 and 3 rejection require additional immunosuppressive treatment. We therefore conclude that quantitative birefringence measurements lack specificity and hence have a low predictive value in the diagnosis of acute rejection, but they have a high sensitivity. It is beyond doubt that quantitative birefringence measurements are of value in assessing the extent of damage to heart tissue as the consequence of rejection, but this is beyond the scope of the present study.

There is a pronounced divergence between cytoimmunological monitoring and quantitative birefringence measurements in relation to endomyocardial biopsy specimens histology. Abnormal cytoimmunological monitoring data (activated lymphocytes) were found for endomyocardial biopsy specimens with grades 2 and 3 rejection, whereas abnormal quantitative birefringence measurement data (lowered values) were found only for endomyocardial biopsy specimens with grades 3 and 4 rejection. This divergence is probably related to the basic processes of acute rejection. Lymphocyte activation is a direct reflection of the effector mechanisms causing acute rejection; myocyte damage is the final result of the graft rejection. Thus mild rejection (grade 2) involves lymphocyte activation without much myocyte necrosis; in resolved or resolving rejection (grade 4) the lymphocyte activation has disappeared leaving a damaged graft in the process of repair. This divergence obviously explains the finding that the combination of cytoimmunological monitoring and quantitative birefringence measurements affords no significant improvement in the diagnosis of acute rejection. The sensitivity in the combined analysis (table 5) is not improved with respect to the segregated calculations (tables 3 and 4), but the specificity reaches values of 0.98 and the predictive value reaches around 0.80 (considering data up to the first episode of acute rejection).

In conclusion, we have documented that cytoimmunological monitoring does offer a useful non-invasive technique in the diagnosis of acute rejection after heart transplantation. We recommend its application, especially until the first episode of acute rejection of the graft or during the first six months after transplantation. Cytoimmunological monitoring may subsequently be useful in the diagnosis of infection. Quantitative birefringence measurements have little tó add to cytoimmunological monitoring in diagnostic terms, but they give valuable background information on the damage to myocytes by the rejection process.

1 Billingham ME. Diagnosis of cardiac rejection by endomyocardial biopsy. J Heart Transplant 1981;1:25-30. Mohanakumar T, Hoshinaga K, Wood NL, et al. Enumeration of transferrin-receptor-expressing lymphocytes as a potential marker for rejection in human cardiac transplan recipients. Transplantation 1986;42:691-4.

3 Lungu O, Reed E, Smith C, et al. Immunological monitoring of heart allograft recipients. Transplant Proc 1987; 19:2548-50.

4 Pelletier LC, Montplaisir S, Pelletier G, et al. Lymphocyte subpopulation monitoring in cyclosporine-treated patients following heart transplantation. Ann Thorac Surg patients following

5 Van Oers MHJ, Van der Heyden AAPAM, Aarden LA. Interleukin 6 (IL-6) in serum and urine of renal transplan recipients. Clin Exp Immunol 1988;71:314-9.

6 Hammer C, Reichenspurner H, Ertel W, et al. Cytological and immunologic monitoring of cyclosporine-treated human heart recipients. J Heart Transplant 1984;3: 228-32.

7 Ertel W, Reichenspurner H, Hammer C, et al. Cytoimmunologic monitoring: a method to reduce biopsy frequency after cardiac transplantation. Transplant Proc 1985;17:204-6.

8 Fieguth H-G, Haverich A, Schäfers H-J, et al. Cytoimmunologic monitoring in early and late acute cardiac rejection. J Heart Transplant 1988;7:95-101.

9 Wijngaard PLJ, Heyn A, Van der Meulen A, et al. Cytommunologic monitoring following heart transplantation. Bibliol Cardiol 1988;43:137-41.

10 Schuurman H-J, Gmelig Meyling FHJ, Wijngaard PLJ, al. Endomyocardial biopsies after heart transplantation presence of immunoglobuline/immune complemen deposits. Transplantation 1989;48:435-8.

11 Schuurman H-J, Jambroes G, Borleffs JCC, et al. Acute humoral rejection after heart transplantation. Transplantation 1988;46:603-5.

12 Baimbridge HW, Chayen J, Bitensky L, et al. Cold cardioplegia or continuous coronary perfusion? $J$ Thorac Carplegia or continuous coronary
diovasc Surg 1977;74:9000-6.

13 Darracott-Cankovic S. Methods for assessing preservation techniques. Invasive methods (enzymatic cytochemical) In: Engelman KM, Vervitsky S, eds. A handbook of clinical cardioplegia. New York: Futura, 1982:43.

14 Chayen J, Bitensky L, Baimbridge HW, et al. Increased myosin orientation during muscle contraction: a measure of cardiac contractibility. Cell Biochem Funct 1985 3:1101-4

15 Darracot-Cankovic S, Wheeldon D, Cory-Pearce $\mathrm{R}$, et al. Biopsy assessment of fifty hearts during transplantation. $J$ Thorac Cardiovasc Surg 1987;93:95-102. 